\title{
In vivo Study of the Osteoregenerative Potential of Polymer Membranes Consisting of Chitosan and Carbon Nanotubes
}

\author{
Marcelo Rodrigues da Cunha ${ }^{a, b *}$, Marianna Carla Alves ${ }^{a}$, Amanda Regina Alves Calegari ${ }^{a}$, \\ Amilton Iatecola ${ }^{a}$, Ewerton Alexandre Galdeano ${ }^{a}$, Talita Lopes Galdeano ${ }^{a}$, Marcelo de Azevedo \\ e Souza Munhoz ${ }^{a, b}$, Ana Maria de Guzzi Plepis ${ }^{b, c}$, Virginia da Conceição Amaro Martins', \\ Marilia Marta Horn ${ }^{b}$ \\ ${ }^{a}$ Departamento de Morfologia e Patologia Básica, Faculdade de Medicina de Jundiai, Rua Francisco \\ Telles, 250, Vila Arens, Cx. Postal 1295, CEP. 13202-550, Jundiai, SP, Brazil \\ ${ }^{b}$ Programa de Pós-graduação Interunidades em Bioengenharia (EESC/FMRP/IQSC), Universidade de \\ São Paulo - USP, Av. Trabalhador São Carlense, 400, CEP: 13566-590, São Carlos, SP, Brazil \\ ${ }^{c}$ Instituto de Quimica de São Carlos - Universidade de São Paulo - USP, Av. Trabalhador São Carlense, \\ 400, CEP: 13566-590, São Carlos, SP, Brazil
}

Received: December 21, 2016; Revised: March 08, 2017; Accepted: April 21, 2017

\begin{abstract}
Biomaterials with the hydroxyapatite and biopolymers such as chitosan derived of crustaceans are is an alternative for bone repair. Carbon nanotubes have been a focus of interest because they can ameliorate the biomechanical properties of biomaterials. The objective of this study was to evaluate these materials in the repair of cranial defects in rats. The animals were divided in groups: without implant (G1), implanted with the chitosan/carbon nanotube membrane (G2), and chitosan/nanotube membrane mineralized with hydroxyapatite (G3). The animals were sacrificed 5 weeks after surgery and the skulls were removed for analysis of the defect area. The results showed absence of chronic inflammatory and little bone neoformation in the defect area of all groups. In G2 and G3 there was lack of reabsorption of the biomaterial that were encapsulated by connective tissue. In conclusion, the biomaterials were biocompatible, but their specific physicochemical properties did not indicate a considerable osteoregenerative capacity.
\end{abstract}

Keywords: Chitosan, Carbon nanotubes, Hydroxyapatite, Polymer blend

\section{Introduction}

Bone repair is a highly complex regenerative process that comprises the interaction of a series of biological events, which are determinant for the restoration of bone tissue ${ }^{1}$. Within this context, there is growing interest in grafts that favor the proliferation of osteogenic cells, thus stimulating bone neoformation associated with local angiogenesis ${ }^{2}$. Autologous and heterologous materials, as well as synthetic implants, used for this purpose have many limitations. Thus, new biocompatible materials have been extensively studied in an attempt to replace previously used materials or to help with bone tissue regeneration. Studies developing new alternative materials for bone repair have focused on natural polymers because of their favorable properties such as biocompatibility.

Among natural polymers, chitosan is widely applicable to the development of materials since it is biocompatible and bioresorbable. Chitosan is a cationic polysaccharide found in the exoskeleton of crustaceans, molluscs and insects, which

* e-mail: cunhamr@hotmail.com is responsible for conferring a structural unit, mechanical stability and resistance to compression forces, similar to the role proteoglycans and glycosaminoglycans play in the fibrillar structure of collagen ${ }^{3}$. Chitosan does not exhibit limitations in terms of quantity, is inexpensive and can be obtained by partial deacetylation of chitin ${ }^{4}$.

Carbon nanotubes (CNTs) are a promising material for application in bone regeneration because of their mechanical and electrical properties ${ }^{5}$. These tubes are composed of $\mathrm{sp}^{2}$ carbon atoms and their length is thousands of times larger than their diameter, a fact making them one of the strongest materials known. On the other hand, the density of CNTs is lower than that of graphite. Comparing their properties to those of other polymers, the high tensile strength, excellent flexibility and low density render CNTs ideal for the production of light materials but with high mechanical resistance ${ }^{6}$. The diameter of single-walled CNTs ranges from 0.7 to $1.5 \mathrm{~nm}$, similar to the size of collagen fibers, a fact that renders these nanotubes a promising material to be used for the nucleation and growth of hydroxyapatite ${ }^{5}$. The interest in CNTs has been growing because of their ability to induce and conduct 
biological events on a nanoscale, in addition to reducing the degradability of biomaterials ${ }^{7}$.

Hydroxyapatite is another widely used product because it permits the phenotypic expression of bone cells. In addition, hydroxyapatite accounts for up to $65 \%$ of the extracellular matrix composition of bone tissue ${ }^{8-10}$. In view of the beneficial properties of hydroxyapatite in bone regeneration, many studies have been conducted to obtain materials with characteristics that can increase the formation of hydroxyapatite in bone tissues ${ }^{11}$.

Venkatesan ${ }^{12}$ described the chitosan and carbon nanotubes scaffolds for use in cell culture, while Wang ${ }^{13}$ relates the preparation and characterization of chitosan/carbon nanotube films. $\mathrm{Im}^{14}$ reported the preparation of chitosan/carbon nanotubes/hydroxyapatite scaffolds using order to osteoblast adhesion. Chen ${ }^{9}$ describes the preparation of chitosan/ multi-walled carbon nanotubes by in situ precipitaton and scaffold croslinking with glutaraldehyde. However, no attemp about in vitro hydroxyapatite mineralization was described, which was the method used in this study and without any crosslink agent.

In an attempt to improve the bioactivity and mechanical properties of materials, in this study, composite materials consisting of chitosan and CNTs coated with an inorganic material (calcium phosphate) by in vitro mineralization were used to induce the growth of hydroxyapatite on their surface. Thus, the scaffold used in this study consists of an organic phase (chitosan) and an inorganic phase (hydroxyapatite), simulating what is found in the human body. However, the ideal material for grafting is still unknown. Therefore, the objective of this study was to analyze the suitability of a chitosan/CNT/ hydroxyapatite composite for the repair of experimental bone defects in rats.

\section{Experimental Procedure}

\subsection{Preparation of the biomaterials}

\section{Raw material}

Chitosan $(\mathrm{CH})$ was prepared in the laboratory from squid pens (Loligo sp.) $)^{15}$. Briefly, the raw material was immerse in a $0.55 \mathrm{~mol} \mathrm{~L}^{-1}(\mathrm{w} / \mathrm{w}) \mathrm{HCl}$ solution at room temperature for $2 \mathrm{~h}$ in order to remove inorganic materials. Then, the precipitate solid was immerse in $0.3 \mathrm{~mol} \mathrm{~L}^{-1}$ (w/w) $\mathrm{NaOH}$ solution at $80^{\circ} \mathrm{C}$ for $1 \mathrm{~h}$ to eliminate proteins. Finally, the last step consist in the alkaline deacetylation of $\beta$-chitin in a $40 \%(\mathrm{w} / \mathrm{w}) \mathrm{NaOH}$ solution for $3 \mathrm{~h}$ at $80^{\circ} \mathrm{C}$, followed by water washes and drying at $40^{\circ} \mathrm{C}$ for 5 days. Chitosan molecular weight (MW) and degree of acetylation (DA) were determined by viscosimetry and condutimetric titration, respectively ${ }^{16}$. DA calculated value was $9.05 \% \pm 0.35$ and MW was $4.28 \times 10^{5}$ Da.

Functionalized multi-walled carbon nanotubes (CN) were kindly provided by Prof. Dr. Benedito dos Santos Lima
Neto, Inorganic and Analytical Group, Instituto de Química de São Carlos, Brasil.

\section{2. $\mathrm{CH}$-CN scaffold preparation}

A $1 \%$ of chitosan solution was obtained by dissolution in acetic acid $1 \%$. After that, $0.25 \mathrm{mg}$ of functionalized $\mathrm{CN}$ was added and solution was then stirred using a mechanic homogenizer followed by homogenization in an ultrasonic bath (Unique USC $1400 \mathrm{~A}$ ) for $60 \mathrm{~min}$. High-energy sonication of $\mathrm{CN}$ is usually necessary to produce uniformly dispersed $\mathrm{CN}$ suspensions. $\mathrm{CH}-\mathrm{CN}$ solution was poured in a Teflon mold $(11 \times 1 \mathrm{~cm})$ and freeze dried during $12 \mathrm{~h}$. Neutralization of acetate in $\mathrm{CH}-\mathrm{CN}$ scaffolds was performed by immersion in $10 \% \mathrm{NaOH}$ solution followed by extensive washing with water and lyophilization in a freeze-dried. Sample was labelled as QN.

\subsection{Scaffolds mineralization}

Mineralization process was performed by alternate soaking method that consists of alternate immersion cycles in two different solutions ${ }^{17}$. Solution 1 was $0.12 \mathrm{~mol} \mathrm{~L}^{-1} \mathrm{CaCl}_{2}$ buffered with $0.05 \mathrm{~mol} \mathrm{~L}^{-1}$ Tris buffer ( $\mathrm{pH}$ 7.4) and Solution 2 was $0.06 \mathrm{~mol} \mathrm{~L}^{-1} \mathrm{Na}_{2} \mathrm{HPO}_{4}$ solution buffered with $0.05 \mathrm{~mol} \mathrm{~L}^{-1}$ Tris buffer ( $\mathrm{pH} 9.0$ ). Scaffolds were placed in $20 \mathrm{~mL}$ of Solution 1 for $60 \mathrm{~min}$, followed by washes in deionized water and subsequent soaking in Solution 2 for $60 \mathrm{~min}$. All experiment was conducted at $37^{\circ} \mathrm{C}$ with two mineralization cycles. Sample were rinsed with water, frozen and lyophilized. Mineralized scaffold was denominated as QNM.

\subsection{Scaffolds characterization}

Termogravimetric Analysis (TG): Thermogravimetric analysis was carried out using a TGA Q50, TA Instruments. Heating was performed in a platinum crucible in synthetic air flow $\left(90 \mathrm{~mL} \mathrm{~min}^{-1}\right)$ at a rate of $10^{\circ} \mathrm{C} \mathrm{min}^{-1}$ from $25^{\circ} \mathrm{C}$ to $800^{\circ} \mathrm{C}$. The sample weight was in the range of $9-10 \mathrm{mg}$.

Scanning Electronic Microscopy (SEM): Samples of about $1 \mathrm{~cm}^{2}$ were coated with a thin layer of gold of $6 \mathrm{~nm}$. The specimens were examined with a ZEISS LEO 440 (Cambridge, England), detector OXFORD (model 7060) LEO 440, with an accelerating voltage of $20 \mathrm{keV}$. Surface and cross-section images were assessed. For pore size determination, the UTHSCSA Image Tool version 3.0 software was used and at least 30 measurements were performed.

Energy Dispersive X-ray Analysis (EDX): $\mathrm{Ca} / \mathrm{P}$ ratio was obtained in an EDX equipment LEO 440, LEO Electron Microscopy Ltd (Cambridge, England), with an Oxford detector Mod. 7060, Oxford Instruments Inc. (Concord, USA) with $133 \mathrm{eV}$ resolution. Standards: $\mathrm{CaCO}_{3}$, quartz, $\mathrm{GaP}$ and Wollas $\left(\mathrm{CaSiO}_{3}\right)$. 


\subsection{Experimental design}

Eighteen Wistar rats, 4 months old and weighing on average $320 \mathrm{~g}$, obtained from the Animal House of the Faculty of Medicine of Jundiaí (FMJ), São Paulo, Brazil, were used. The study was approved by the Ethics Committee on Animal Experimentation of FMJ (Protocol 90/2015).

The animals were anesthetized by intramuscular gluteal injection of ketamine and xylazine (proportion of 1:1) at a dose of $0.10 \mathrm{mg} / 100 \mathrm{~g}$ body weight. Next, a defect measuring 5 $\mathrm{mm}$ in diameter and $1 \mathrm{~mm}$ in thickness was created surgically in the left parietal bone of the skullcap, preserving the dura mater. After surgery, the periosteum, soft tissues and skin were repositioned and sutured with 6.0 silk suture, and the animals received routine postoperative care ${ }^{18}$.

The animals were divided into three groups: G1 (unfilled cranial defect); G2 (cranial defect filled with the chitosan/CN membrane - QN); G3 (cranial defect filled with the chitosan/ $\mathrm{CN}$ membrane mineralized with calcium phosphate - QNM). The animals were euthanized 5 weeks after surgery with an overdose of the anesthetic followed by the inhalation of carbon dioxide. The skullcaps of the animals were removed and sent for macroscopic and radiological analysis. Tissue sections were obtained from the defect area, submitted to routine histological procedures, and stained with Masson's trichrome and Picrosirius red. The volume of newly formed bone was measured using the principle of Delesse ${ }^{19}$. The results were analyzed with the BioEstat program using ANOVA followed by the Tukey test. A level of significance of $\mathrm{p}<0.05$ was adopted.

\section{Results}

\subsection{Characterization of biomaterials}

Thermal stability and quantification of mineralization were assessed by thermogravimetric (TG) analysis, in which three stages of weight loss were observed (Figure 1). The first one between 25 and $200^{\circ} \mathrm{C}$ is associated with the release of water present in scaffolds. The second, in the range $200-400^{\circ} \mathrm{C}$, is due to the degradation of the polymer molecule and the third one, that occurs in the range $400-650^{\circ} \mathrm{C}$, is related to the combustion of the residual components. Residues at $750^{\circ} \mathrm{C}$ were obtained and allowed to determine the inorganic material content in the scaffolds. Hydroxyapatite (product desirable in mineralization process) is stable at this temperature ${ }^{20}$.

The value for residue at $750^{\circ} \mathrm{C}$ for QNM was $16.4 \%$. For QN no residue was observed at this temperature.

Bioactivity of scaffolds can be evaluated by studying the calcium salts deposition on its surface and internal structure. SEM images of the surface of freeze-dried scaffolds before and after mineralization cycle are shown in Figure 2. In addition, QN scaffold (Figure 2A) has pores of around $52.5 \pm 3.2 \mu \mathrm{m}$ that allows vascularization and are suitable for fibroblasts infiltration ${ }^{21}$. Surface of the mineralized scaffold

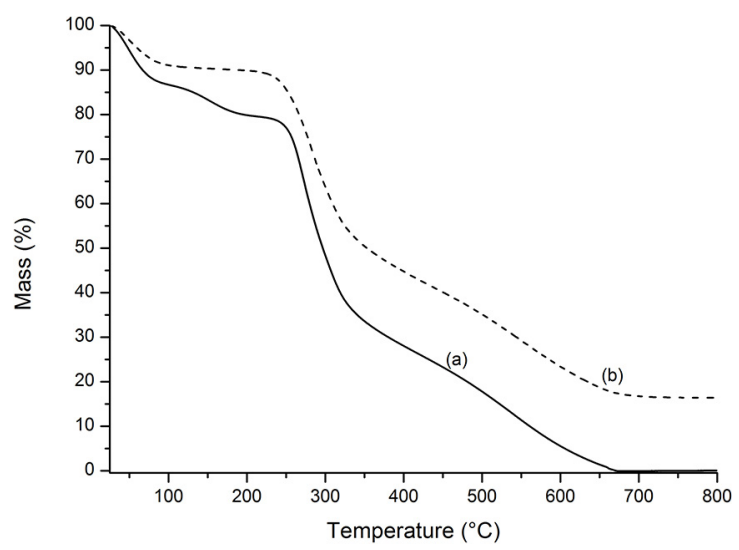

Figure 1. TG curves of (a) QN and (b) QNM.

shows mineral deposits constituted of few apatite particles after two mieralization cycles (Figure 2B). Mineralization process decreases both the quantity and the pore size, as QNM had value of pore size of $39.2 \pm 2.8 \mu \mathrm{m}$.

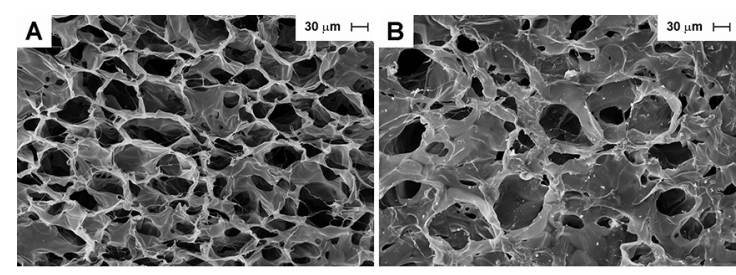

Figure 2. SEM images of (A) QN and (B) QNM. Magnification of $500 \mathrm{x}$.

At a magnification of $20.000 \mathrm{x}$ (Figure 3) the mineral deposits appeared to be spherical in shape and have a size of $0.73 \pm 0.10 \mu \mathrm{m}$ in diameter.

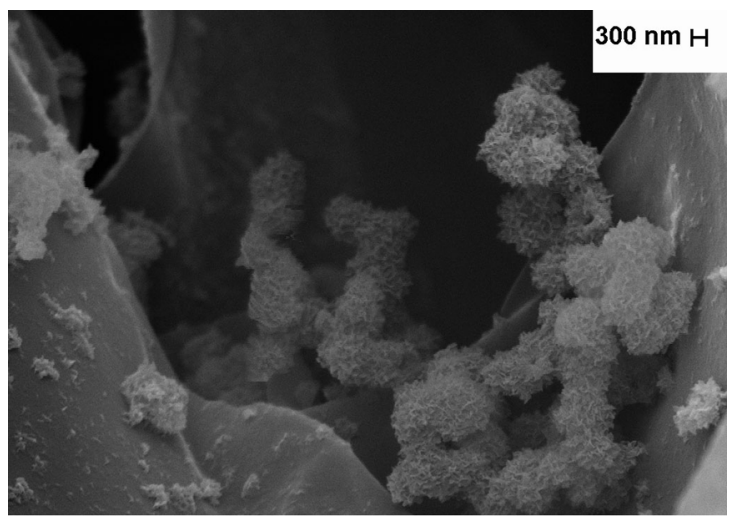

Figure 3. SEM images of QNM. Magnification of $20.000 \mathrm{x}$.

EDS analysis (Figure 4) indicated that HA deposits consist of calcium, phosphorous, and oxygen and no other elements were observed, confirming the high purity of the calcium phosphate. The $\mathrm{Ca} / \mathrm{P}$ molar ratio for QNM was $1.44 \pm 0.03$ that indicates a calcium deficient hydroxyapatite. 


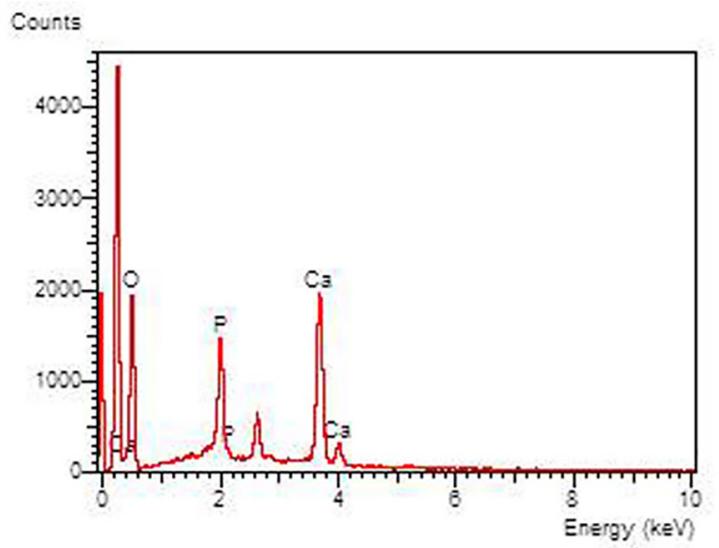

Figure 4. EDS spectrum of QNM.

\subsection{Macroscopic and radiological analysis}

Good healing of soft tissues was observed in the defect area and there were no clinical indications of complications related to chronic infections. Persistence of the bone defect was evident since no total repair occurred. In the grafted groups (G2 and G3), the biomaterials were present in the defect area and were not reabsorbed within the experimental period established in this study. The experimentally induced bone defect characterized by radiodense regular borders and a radiolucent central image was observed in all radiographed specimens. A discrete radiopaque image was noted in G2, which was due to the presence of the chitosan membrane. This density of the material was more visible in G3 because of mineralization of the membrane (Figure 5).
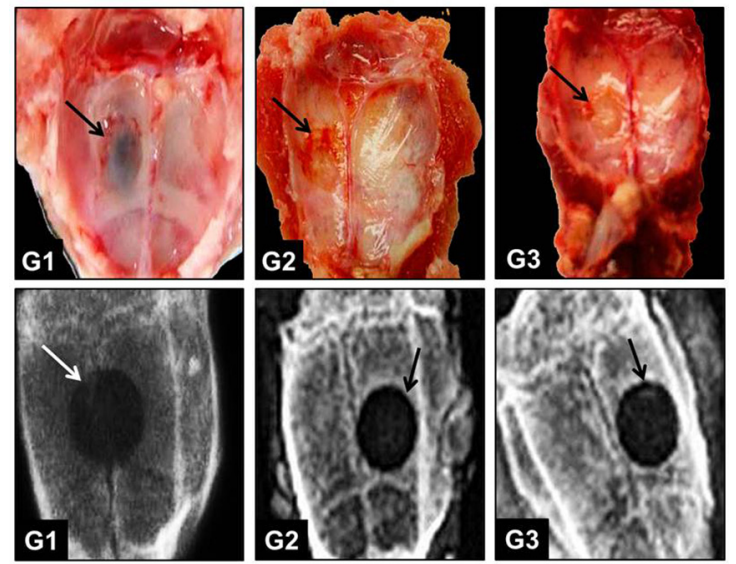

Figure 5. Inferior view after removal of the skullcap in the three groups studied (G1, G2, and G3). Note the defect area (arrow) without alterations and the presence of the implanted biomembranes in G2 and G3. Radiographic image of the superior view of the skullcap in the three groups studied (G1, G2, and G3). Observe the preservation of cranial anatomy and persistence of the experimentally induced bone defect. In G2 and G3, a radiopaque image is evident in the center of the defect, indicating the presence of the implanted biomembranes which were not reabsorbed during the experimental period.

\subsection{Histological analysis}

No significant bone repair was observed in the experimentally induced defects of the groups studied (G1, G2, and G3), considering the predominant proliferation of connective tissue (Figure 6). Collagen fiber organization (data not shown), as well as discrete bone neoformation from the margins of the defect, occurred in all groups. This young bone exhibited immature features consisting of collagen fiber disorganization and the irregular arrangement of oval lacunae containing osteocytes. In G2 and G3, the connective tissue formed encapsulated the implanted membrane and was also found at the bone/graft interface, decharacterizing osseointegration of the membrane. The formation of small bone areas at the bone/graft interface was observed in G3. In addition, the lack of reabsorption of the membrane was noted in the grafted groups throughout the experimental period (Figure 6).

\subsection{Morphometric analysis}

The mean ( \pm standard deviation) percentage of newly formed bone was $7.75 \pm 1.5,8.5 \pm 1.2$ and $9.5 \pm 1.2$ in G1, G2 and G3, respectively. Statistical analysis showed no difference $(p<0.05)$ between groups.

\section{Discussion}

Biocompatibility is an important factor for the use of biomaterials and is related to the capacity of a material to elicit an adequate response in the host without immunological rejection ${ }^{2}$. Researchers studied the in vitro production of osteocalcin by osteoblasts and in vivo bone formation using a composite made from chitosan and hydroxyapatite as support. The authors demonstrated the biocompatibility and osteoconductivity of this composite in rat cranial defects and suggested its indication for use in tissue engineering ${ }^{22}$. In the present study, the biomembranes consisting of calcium phosphate, chitosan and CNTs were also biocompatible as demonstrated by the macroscopic and radiographic absence of vascular necrosis characterized by acellular tissue and chronic inflammatory and infectious processes at the recipient site, signs that indicate a foreign body reaction.

Defects of $5 \mathrm{~mm}$ can be considered critical since no bone total formation was observed during the experimental period in the control animals (G1), showing that these defects require an osteoregenerative stimulation by the use of synthetic or natural grafts. Previous researches used defects of $5 \mathrm{~mm}^{23-25}$. The rat skullcap has two parietal bones (right and left) separated by a sagittal suture of medium (middle) location. In this study, the bone defect occupied the entire width of the parietal bone where the biomaterial was grafted and a thin border of the original bone was preserved which ensured the stability of biomaterial graft. 


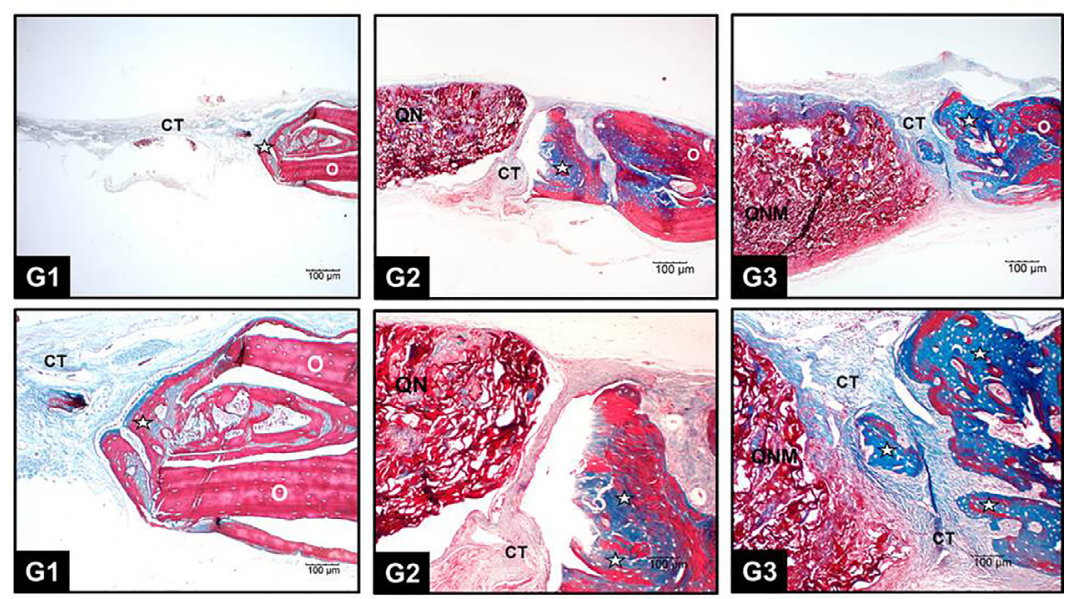

Figure 6. Photomicrograph of the defect area in animals of groups 1, 2 and 3 (G1, G2, and G3) at $4 \mathrm{x}$ and 10x magnification. Using Masson's trichrome, note the formation of new bone $\left({ }^{*}\right)$ stained blue from the margins of the original bone $(\mathrm{O})$ stained red and the predominance of connective tissue (CT) in the cranial defect. Connective tissue is present between the bone and implanted membranes (QN, QNM) in the bone defect.

The ideal graft should have various characteristics; most importantly, the graft should be non-immunogenic ${ }^{26}$. The success of the implantation of biomaterials is related to the suitability of the recipient site that should permit bone growth and stability of the implant in the absence of macromovements that can interfere with the consolidation process $^{27}$. In agreement with these statements, in the present study, macroscopic and radiological analysis revealed maintenance of the membranes in the center of the defect in $\mathrm{G} 2$ and $\mathrm{G} 3$. A radiolucent image was observed inside the bone defect in G1, which did not receive the implant. This radiological feature is due to the proliferation of abundant connective tissue or the formation of small amounts of new bone. The development of connective tissue in the center of the defect and of immature young bone is observed at the beginning of any repair process. The radiopacity of the latter is insufficient to be detected upon radiographic examination. Thus, the radiological results observed were similar to those reported in the literature ${ }^{28,29}$. A denser image was noted in the center of the cranial defect in $\mathrm{G} 2$ and $\mathrm{G} 3$, which was due to the presence of the biomembranes that were not reabsorbed within the experimental period established in this study.

The success of any type of implant or graft does not only depend on the biocompatibility and type of the material. Features such as dimensions, surface properties, characteristics of the recipient site, trauma during surgery and movement of the bone/implant interface should be taken into consideration when the efficacy of osseointegration of the material is determined ${ }^{30}$. Histological analysis of the defect areas in the groups receiving the membranes showed little bone formation from the margins of the defect, the absence of bone encasement of the graft, and the marked presence of connective tissue adjacent to the membrane and at the bone/implant interface, decharacterizing osseointegration of the biomaterial as reported in other research ${ }^{31}$. In addition to the direct contact between the bone and implant known as osseointegration, there is another type of Integration of the bone with fibrous tissue, known as fibro-osseous integration, indicating the absence of total osseointegration of the biomaterial used ${ }^{32}$. This was the most observed histological feature in $\mathrm{G} 2$ and $\mathrm{G} 3$.

Surface contamination of the grafted material to be a determinant factor for the successful integration of an implant into organic tissues. The presence of residues on the surface of the biomaterial can alter its chemical composition. Researches confirmed this fact by demonstrating the influence of toxic agents derived from biomaterials on functionality and cell viability after implantation into the recipient site, which can cause cell death due to the absence of metabolic activity, structural disintegration (cell membrane) and cell lysis ${ }^{31}$. Other authors emphasize that reabsorption is a desirable characteristic of biomaterials whose degradation occurs simultaneously with the replacement of newly formed bone ${ }^{33}$. In contrast, in the present study the membranes remained intact (G2 and G3), acting as a barrier to bone growth.

Besides the degree of degradation of the implanted biomaterial, porosity is also extremely important since bone cell growth should occur through the pores of the implanted material, promoting osseointegration ${ }^{34}$ Porous scaffold facilitates cell migration, proliferation and differentiation, allowing the transportation of nutrients and oxygen within the structure ${ }^{35}$. Another relevant factor is the interconnectivity between pores to permit the invasion of osteoblasts and cell attachment. In addition, these connections between pores allow blood circulation, the exchange of body fluids and the diffusion of ions. Unconnected pores do not participate in these physiological events that are necessary for regeneration ${ }^{36}$. In the present study, QN scaffold has 
pores of around $52.5 \pm 3.2 \mu \mathrm{m}$. The porosity of the grafted membranes in G2 and G3 was probably not ideal to permit bone cell and vascular proliferation, since the newly formed bone was concentrated only at the margins of the defect, while interposed fibrotic tissue predominated in the center. Furthermore, in G3, the amount of hydroxyapatite crystals may not have been sufficient to stimulate osteogenesis. In this case, it should be considered that the surface of the mineralized scaffold used in this research has mineral deposits consisting of a few apatite particles and the mineralization process decreases both the quantity and the size of the pores since the QNM had a pore size of $39.2 \pm 2.8 \mu \mathrm{m}$. This alteration of the pores may compromise cell and vascular proliferation. Thus, further studies on the absorption, porosity and quantity of mineralization of materials are necessary. Promising results of bone regeneration using natural polymers such as collagen, chitosan or a combination of both have been reported in the literature; however, these natural polymers differed in their manufacturing characteristics and chemical processes, factors that directly interfere with the regenerative properties of these materials ${ }^{37}$.

The suitability of chitosan polymers as composites requires further investigation considering that in the present study no promising results were obtained regarding the volume of newly formed bone in the defect area of the animals. In this respect, the search for the ideal graft biomaterial continues to be a challenge since osseointegration and acceptable bone repair depend not only on the physiochemical composition and three-dimensional arrangement of biomaterials, but also on the morphological characteristics of the recipient site.

\section{Conclusions}

The biomembranes used exhibited biocompatibility with the recipient bone, but did not represent a considerable osteoregenerative factor for the stimulation of osseointegration or bone repair during the experimental period. However, this finding should not discourage further research on the application of chitosan in regenerative therapies since other studies have reported favorable results of the use of chitosan in tissue healing. Furthermore, external factors such as the type of bone, local muscle action and postoperative cell reaction may also interfere with the process of bone regeneration.

\section{Acknowledgments}

Prof. Dr. Benedito dos Santos Lima Neto and this study was supported by the CNPq, Institute of Chemistry - USP (São Carlos), NAPED/FMJ and PNPD/Capes.

\section{References}

1. Agarwal R, García AJ. Biomaterials strategies for engineering implants for enhanced osseointegration and bone repair. Advanced Drug Delivery Reviews. 2015;94:53-62.
2. Ratner BD, Hoffman AS, Schoen FJ, Lemons JE. Biomaterials Science. An Introduction to Materials in Medicine. $3^{\text {rd }}$ ed. Oxford: Academic Press; 2013.

3. LogithKumar R, KeshavNarayan A, Dhivya S, Chawla A, Saravanan S, Selvamurugan N. A review of chitosan and its derivatives in bone tissue engineering. Carbohydrate Polymers. 2016;151:172-188.

4. Horn MM, Martins VCA, Plepis AMG. Influence of collagen addition on the thermal and morphological properties of chitosan/xanthan hydrogels. International Journal of Biological Macromolecules. 2015;80:225-230.

5. Eatemadi A, Daraee H, Karimkhanloo H, Kouhi M, Zarghami $\mathrm{N}$, Akbarzadeh A, et al. Carbon nanotubes: properties, synthesis, purification and medical applications. Nanoscale Research Letters. 2014;9(1):393.

6. Aryaied A, Jayatissa AH, Jayasuriya AC. Mechanical and biological properties of chitosan/carbon nanotube nanocomposite films. Journal of Biomedical Materials Research Part A. 2014;102(8):2704-2712.

7. Newman P, Minett A, Ellis-Behnke R, Zreiqat H. Carbon nanotubes: Their potential and pitfalls for bone tissue regeneration and engineering. Nanomedicine: Nanotechnology, Biology and Medicine. 2013;9(8):1139-1158.

8. Cunha MR, Santos Jr AR, Goissis G, Genari SC. Implants of polyanionic collagen matrix in bone defects of ovariectomized rats. Journal of Materials Science: Materials in Medicine. 2008;19(3):1341-1348.

9. Chen L, Hu J, Shen X, Tong H. Synthesis and characterization of chitosan-multiwalled carbon nanotubes/hydroxyapatite nanocomposites for bone tissue engineering. Journal of Materials Science: Materials in Medicine. 2013;24(8):1843-1851.

10. Zimmermann G, Moghaddam A. Allograft bone matrix versus synthetic bone graft substitutes. Injury. 2011;42(Suppl. 2):S16-S21.

11. Tavakol S, Nikpour MR, Amani A, Soltani M, Rabiee SM, Rezayat $\mathrm{SM}$, et al. Bone regeneration based on nano-hydroxyapatite and hydroxyapatite/chitosan nanocomposites: an in vitro and in vivo comparative study. Journal of Nanoparticles Research. 2013;15(1):1-16.

12. Venkatesan J, Ryu B, Sudha PN, Kim SK. Preparation and characterization of chitosan-carbon nanotube scaffolds for bone tissue engineering. International Journal of Biological Macromolecules. 2012;50(2):393-402.

13. Wang SF, Shen L, Zhang WD, Tong YJ. Preparation and Mechanical Properties of Chitosan/Carbon Nanotubes Composites. Biomacromolecule. 2005;6(6):3067-3072.

14. Im O, Li J, Wang M, Zhang LG, Keidar M. Biomimetic threedimensional nanocrystalline hydroxyapatite and magnetically synthesized single-walled carbon nanotube chitosan nanocomposite for bone regeneration. International Journal of Nanomedicine. 2012;7:2087-2099.

15. Horn MM, Martins VCA, Plepis AMG. Interaction of anionic collagen with chitosan: Effect on thermal and morphological characteristics. Carbohydrate Polymers. 2009;77(2):239-243. 
16. Raymond L, Morin FG, Marchessault RH. Degree of deacetylation of chitosan using conductometric titration and solid-state NMR. Carbohydrate Research. 1993;246(1):331-336.

17. Kretlow JD, Mikos AG. Review: mineralization of synthetic polymer scaffolds for bone tissue engineering. Tissue Engineering. 2007;13(5):927-938.

18. Cunha MR, Menezes FA, Santos GR, Pinto CAL, Barraviera B, Martins VCA, et al. Hydroxyapatite and a New Fibrin Sealant Derived from Snake Venom as Scaffold to Treatment of Cranial Defects in Rats. Materials Research. 2015;18(1):196-203.

19. Mandarim-de-Lacerda CA. Whats is the interest of normal and pathological morphological to be quantitative? The exemple of the stereology. Brazilian Journal of Morphological Sciences. 1999;16(2):131-139.

20. Lv Y, Huang H, Yang B, Liu H, Li Y, Wang J. A robust $\mathrm{pH}$-sensitive drug carrier: Aqueous micelles mineralized by calcium phosphate based on chitosan. Carbohydrate Polymers. 2014;111:101-107.

21. Tanase CE, Sartoris A, Popa MI, Verestiuc L, Unger RE, Kirkpatrick CJ. In vitro evaluation of biomimetic chitosancalcium phosphate scaffolds with potential application in bone tissue engineering. Biomedical Materials. 2013;8(2):25002.

22. Chesnutt BM, Yuan Y, Buddington K, Haggard WO, Bumgardner JD. Composite chitosan/nano-hydroxyapatite scaffolds induce osteocalcin production by osteoblasts in vitro and support bone formation in vivo. Tissue Engineering. Part A. 2009;15(9):25712589 .

23. Potijanyakul P, Sattayasansakul W, Pongpanich S, Leepong N, Kintarak S. Effects of enamel matrix derivative on bioactive glass in rat calvarium defects. Journal of Oral Implantology. 2010;36(3):195-204.

24. Hirata HH, Munhoz MA, Plepis AM, Martins VC, Santos GR, Galdeano EA, et al. Feasibility study of collagen membranes derived from bovine pericardium and intestinal serosa for the repair of cranial defects in ovariectomised rats. Injury. 2015;46(7):1215-1222.

25. Rojbani H, Nyan M, Ohya K, Kasugai S. Evaluation of the osteoconductivity of $\alpha$-tricalcium phosphate, $\beta$-tricalcium phosphate, and hydroxyapatite combined with or without simvastatin in rat calvarial defect. Journal of Biomedical Materials Research Part A. 2011;98(4):488-498.
26. Billström GH, Blom AW, Larsson S, Beswick AD. Application of scaffolds for bone regeneration strategies: current trends and future directions. Injury. 2013;44 Suppl 1:S28-33.

27. Shors EC. Coralline bone graft substitutes. Orthopedic Clinics of North America. 1999;30(4):599-613.

28. Hirata MM, Munhoz MA, Plepis AMG, Martins VCA, Santos GR, Galdeano EA, et al. Feasibility study of collagen membranes derived form bovine pericardium and intestinal serosa for the repair of cranial defects in ovariectomised rats. Injury. 2015;46(7):1215-1222.

29. da Cunha MR, Gushiken VO, Mardegan Issa JP, Iatecola A, Pettian M, Santos AR Jr. Osteoconductive capacity of hidroxiapatite implanted into the skull of diabectis. Journal of Craniofacial Surgery. 2011;22(6):2048-2052.

30. Dubois JC, Souchier C, Couble ML, Exbrayat P, Lissac M. An image analysis method for the study of cell adesion to biomaterials. Biomaterials 1999;20(19):1841-1849.

31. Konig Jr B. Implantology and Osseointegration: how to research in ceramics, clinical applications and other materials. São Paulo: Rocca; 2010.

32. Legeros RZ, Craig RG. Strategies to affect bone remodeling: Osteointegration. Journal of Bone and Mineral Research. 1993;8 Suppl 2:S583-S596.

33. Henkel J, Woodruff MA, Epari DR, Steck D, Glatt V, Dickinson IC, et al. Bone Regeneration Based on Tissue Engineering Conceptions - A 21st Century Perspective. Bone Research. 2013;1(3):216-248.

34. Hannink G, Arts JJ. Bioresorbability, porosity and mechanical strength of bone substitutes: what is optimal for bone regeneration? Injury. 2011;42 Suppl 2:S22-25.

35. Velasco MA, Narváez-Tovar CA, Garzón-Alvarado DA. Design, Materials, and Mechanobiology of Biodegradable Scaffolds for Bone Tissue Engineering. BioMed Research International. 2015;2015:729076.

36. Sopyan I, Mel M, Ramesh S, Khalid KA. Porous hydroxyapatite for artificial bone applications. Science and Technology of Advanced Materials. 2007;8(1-2):116-123.

37. Anastasia L, Rota P, Anastasia M, Allevi P. Chemical structure, biosynthesis and synthesis of free and glycosylated pyridinolines formed by cross-link of bone and synovium collagen. Organic and Biomolecular Chemistry. 2013;11(35):5747-5771. 